\title{
Terahertz Nonlinear Optics in Semiconductors
}

\section{Turchinovich, Dmitry; Hvam, Jørn Märcher; Hoffmann, Matthias C.}

\section{Published in:}

Proceedings of the 38th International Conference on Infrared, Millimeter and Terahertz Waves IRMMW-THz 2013

Link to article, DOI:

10.1109/IRMMW-THz.2013.6665865

Publication date:

2013

Link back to DTU Orbit

Citation (APA):

Turchinovich, D., Hvam, J. M., \& Hoffmann, M. C. (2013). Terahertz Nonlinear Optics in Semiconductors. In Proceedings of the 38th International Conference on Infrared, Millimeter and Terahertz Waves IRMMW-THz 2013 IEEE. https://doi.org/10.1109/IRMMW-THz.2013.6665865

\section{General rights}

Copyright and moral rights for the publications made accessible in the public portal are retained by the authors and/or other copyright owners and it is a condition of accessing publications that users recognise and abide by the legal requirements associated with these rights.

- Users may download and print one copy of any publication from the public portal for the purpose of private study or research.

- You may not further distribute the material or use it for any profit-making activity or commercial gain

- You may freely distribute the URL identifying the publication in the public portal

If you believe that this document breaches copyright please contact us providing details, and we will remove access to the work immediately and investigate your claim 


\title{
Terahertz Nonlinear Optics in Semiconductors
}

\author{
Dmitry Turchinovich ${ }^{\mathrm{a}, \mathrm{b}}$, Jørn M. Hvam ${ }^{\mathrm{b}}$, and Matthias C. Hoffmann ${ }^{\mathrm{c}}$ \\ ${ }^{a}$ Max Planck Institute for Polymer Research, 55128 Mainz, Germany \\ ${ }^{\mathrm{b}}$ Technical University of Denmark, 2800 Lyngby, Denmark \\ 'SLAC Linear Accelerator Laboratory, Menlo Park, California, U.S.A
}

\begin{abstract}
We demonstrate the nonlinear optical effects - selfphase modulation and saturable absorption of a single-cycle $\mathbf{T H z}$ pulse in a semiconductor. Resulting from THz-induced modulation of Drude plasma, these nonlinear optical effects, in particular, lead to self-shortening and nonlinear spectral breathing of a single-cycle $\mathrm{THz}$ pulse in a semiconductor.
\end{abstract}

\section{INTRODUCTION AND BACKGROUND}

$\mathrm{W}$ E present the nonlinear optical effects such as saturable absorption (SA) and self-phase modulation (SPM) of a single-cycle $\mathrm{THz}$ pulse in a doped semiconductor. The nonlinearity arises from the electron plasma response to the ponderomotive potential of the strong-field $\mathrm{THz}$ pulse, which leads to electron heating and intervalley electron scattering. This produces ultrafast modification of electron plasma frequency via increase of average electron effective mass (see Fig. 1). The complex-valued dielectric function $\hat{\epsilon}(\omega)$ of a semiconductor in the presence of free carriers is described by a well-known Drude model:

$$
\hat{\varepsilon}=\left(n+\frac{i \alpha c}{2 \omega}\right)^{2}=\varepsilon_{d c}-\frac{\omega_{p}^{2}}{\omega^{2}-i \omega / \tau}
$$

where $n$ and $\alpha$ are frequency-dependent refractive index and power absorption coefficient, $\omega_{\mathrm{p}}$ - plasma frequency, and $\tau$ is electron momentum scattering rate. Plasma frequency

$$
\omega_{p}=\left(N e^{2} / \varepsilon_{0} m\right)^{1 / 2}
$$

where $N$ is free carrier density, $e$ is elementary charge, $\epsilon_{0}$ is the vacuum permittivity, and $m$ is the effective mass.

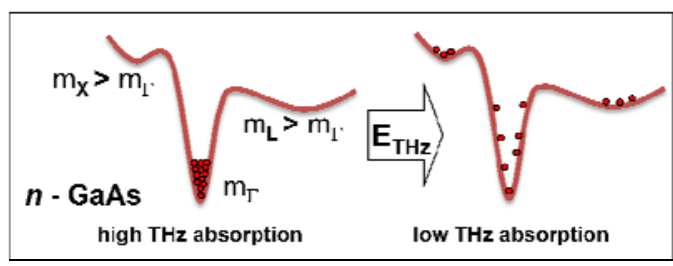

Fig. 1. Mechanism of $\mathrm{THz}$ nonlinearity in doped semiconductors by carrier heating in $\mathrm{THz}$ field.

It is apparent from eqs. (1) and (2), that at constant carrier density, the increase of the electron effective mass leads to reduction of the plasma frequency, and hence to the reduction of absorption coefficient and to the change in the refractive index. The heating of low-effective-mass $\Gamma$-valley electrons in the ponderomotive potential of a strong-field $\mathrm{THz}$ pulse leads to intervalley scattering in the energy and momentum space -
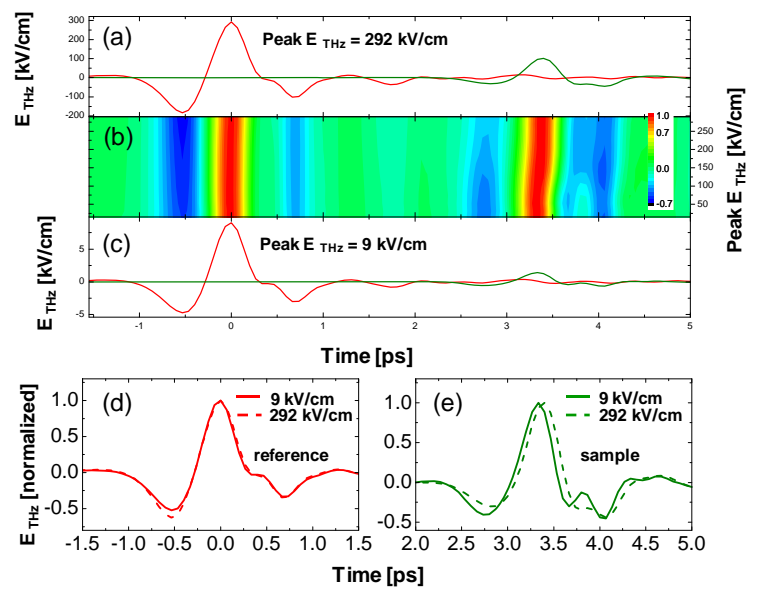

Fig. 2. $\mathrm{THz}$ SPM in $\mathrm{n}-\mathrm{GaAs}$ in the time domain. Peak $\mathrm{E}_{\mathrm{THz}}$ of reference THz pulse in (a) is $292 \mathrm{kV} / \mathrm{cm}$, and in (c) is $9 \mathrm{kV} / \mathrm{cm}$. (b) Reference and sample pulses normalized to their maxima, for the whole range of peak $E_{T H z}$ of $9-292 \mathrm{kV} / \mathrm{cm}$. (d,e) Normalized reference (d) and sample (e) pulses for the cases of peak $E_{T H z}$ of 9 $\mathrm{kV} / \mathrm{cm}$ and $292 \mathrm{kV} / \mathrm{cm}$, demonstrating the SPM.

the population of higher-energy satellite valley (L- and Xvalleys) featuring larger effective masses. This naturally leads to an increase in the average effective mass of the electron population, and hence to a reduction of the plasma frequency of a semiconductor. The principle of such as THz-induced nonlinearity in a doped semiconductor is illustrated in Fig. 1. As a result, the complex dielectric function of a semiconductor undergoes dramatic modification during the interaction with the $\mathrm{THz}$ pulse, leading to its nonlinear propagation. In this study the n-GaAs was chosen as a sample, however any semiconductor with complex band structure will produce the $\mathrm{THz}$ nonlinearity via the same mechanism.

\section{RESULTS}

In our studies we have used the nonlinear $\mathrm{THz}$ time-domain spectroscopy (NL THz-TDS) based on the strong-field $\mathrm{THz}$ emitter - the tilted pulse front pumped lithium niobate [1]. Our experiments were performed in a traditional transmission configuration, and for each measurement two $\mathrm{THz}$ waveforms - reference (THz propagation through vacuum) and sample ( $\mathrm{THz}$ propagation through vacuum and sample) were measured. The $\mathrm{THz}$ peak field strength was controllably attenuated in the range $9-292 \mathrm{kV} / \mathrm{cm}$ using crossed wire-grid polarizers. 
The optical nonlinearities are observed directly in the time domain (see Fig. 2), and characterized in the frequency domain (Fig. 3).
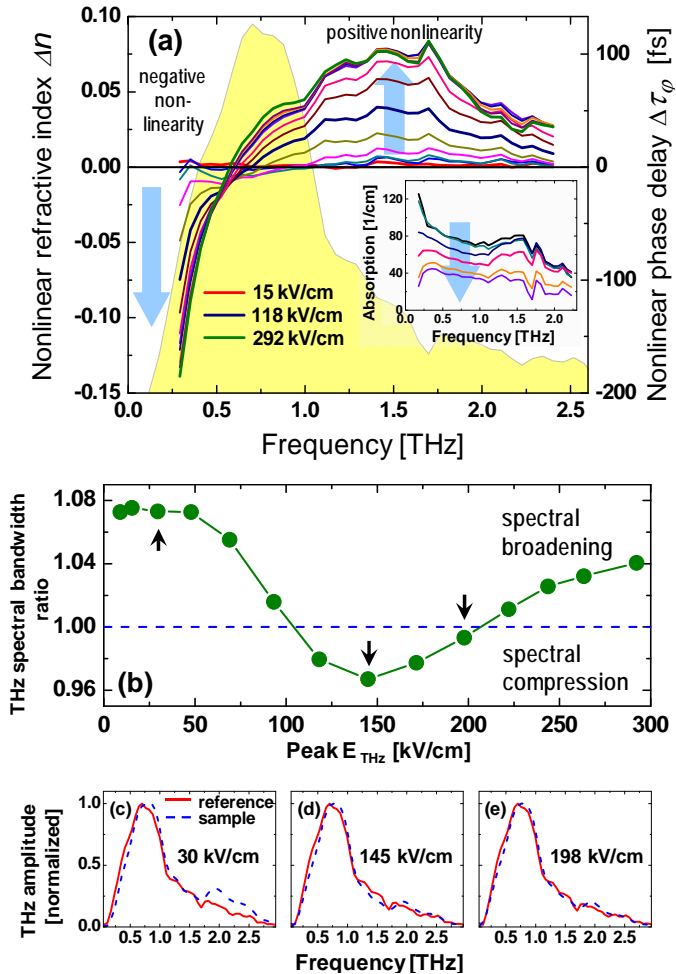

Fig. 3. (a) THz SPM and saturable absorption in n-GaAs in spectral domain. Nonlinear contribution to refractive index $\Delta n$, and corresponding nonlinear phase delay as a function of frequency and peak $E_{T H z}$. Inset: spectrally resolved saturable absorption. The amplitude spectrum of the reference $\mathrm{THz}$ pulse is shown as a background. (b-e) Nonlinear spectral broadening and compression of a THz pulse in $n$-GaAs. (b)The ratio of effective bandwidths of the sample and reference pulses, as a function of peak $E_{\mathrm{THz}}$. (c-e) Examples of spectral dynamics at selected peak $\mathrm{THz}$ field values.

In particular, we present the effects of self-shortening of a single-cycle $\mathrm{THz}$ pulse [2], and $\mathrm{THz}$ self-phase modulation (SPM) observed on a single-cycle waveform, with sub-cycle time resolution [3], as shown in Fig. 2. The THz SPM, similar to the nonlinear optics demonstrated in the infrared and visible spectral ranges, results in nonlinear spectral breathing of the $\mathrm{THz}$ waveform. Further, we have found that the sign of the THz-range refractive index nonlinearity in doped semiconductor can be both positive and negative. In fact, we have discovered the co-existence of positive and negative refractive index nonlinearity within the spectral bandwidth of a single-cycle $\mathrm{THz}$ waveform [3]. This is quite a unique situation in nonlinear optics, though hardly unexpected given an inherently ultrabroadband nature of any single-cycle waveform.

All our findings, including the co-existence of index nonlinearity of different signs within the spectrum of the same $\mathrm{THz}$ waveform, can be well described within the Drude plasma model. For example, we have found that the point of zero $\mathrm{THz}$ refractive index nonlinearity is defined by (but is not equal to) the electron momentum relaxation rate $\tau$ in the semiconductor, as a result of the transition between lowfrequency Hagen-Rubens regime featuring a larger refractive index, and higher frequency conductivity featuring a reduced values of $n$ in the presence of free carriers.

In summary, the effects of saturable absorption, self-phase modulation, pulse self-shortening, and nonlinear spectral breathing were demonstrated in nonlinear propagation of a single-cycle $\mathrm{THz}$ waveform through a doped semiconductor. The THz nonlinearity is caused by the nonlinear response of the free carriers to the strong $\mathrm{THz}$ fields, leading in particular to inter-valley scattering.

\section{REFERENCES}

[1] K.-L. Yeh, M. C. Hoffmann, J. Hebling, and K. A. Nelson, Appl. Phys. Lett. 90, 171121 (2007).

[2] M. C. Hoffmann and D. Turchinovich, Appl. Phys. Lett., 96, 151110 (2010).

[3] D. Turchinovich, J. M. Hvam, and M. C. Hoffmann, Phys. Rev. B 85, 201304(R) (2012) 